\title{
1,8-Hydrogen Atom Transfer Promoted by N-Radicals in $(1<4)$-O -Disaccharide Models
}

\author{
Elisa I. León, ${ }^{[a]}$ Ángeles Martín,*[a] Inés Pérez-Martín,*[a] and Ernesto Suárez ${ }^{[a]}$
}

Keywords: Carbohydrates / Radical reactions / Hydrogen transfer / Radicals

The nitrogen-centered radical generated by reaction of an $\mathrm{N}$-sulfonamidate, attached to C-6 of a hexopyranose in a disaccharide model, with (diacetoxyiodo)benzene (DIB) and iodine undergoes a novel regio- and stereoselective intramolecular 1,8-hydrogen atom transfer (HAT) reaction to promote the functionalization of remote positions.

\section{Introduction}

Intramolecular hydrogen atom transfer (HAT) is one of the most interesting radical processes, because it allows the completely regioselective functionalization of remote positions. ${ }^{[1]}$ In recent years our laboratory has devoted its attention to the application of this process, promoted by $\mathrm{O}^{[2]}$ or $\mathrm{N}$-radicals, ${ }^{[3]}$ to sugar templates. In these studies, intramolecular 1,5- and 1,6-HAT reactions were initiated by alkoxyl or aminyl radicals derived from an alcohol or suitably protected amine, respectively, by treatment with a hypervalent iodine reagent in the presence of iodine. The results demonstrated the synthetic potential of this methodology for the preparation of different five- or six-membered heterocyclic compounds. As a consequence of these results, and considering the lack of reported examples in sugars, ${ }^{[4]}$ we envisioned that an intramolecular HAT reaction via a higherthan-seven-membered transition state might be possible. The results of these investigations revealed a successful and highly unusual 1,8-HAT reaction promoted by an alkoxyl radical. ${ }^{[5]}$ This process occurs between the two monosaccharide units in a $(1<4)-O$-disaccharide suitably substituted to meet the required geometrical and stereoelectronic conditions via a nine-membered transition state (Scheme 1). In this process, if a 1,3,5-trioxocane ring $\mathbf{I}$ could be formed in a stable boat-chair conformation, the abstraction would occur preferentially at C-5', whereas if the process is energetically disfavored, in conformations similar to a boatboat or crown ether, the abstraction would take place mainly at C-1'.

[a] Instituto de Productos Naturales y Agrobiología del C.S.I.C., Carretera de la Esperanza 3, 38206 La Laguna, Tenerife, Spain Fax: +34-922-260-135

E-mail: angelesmartin@ipna.csic.es ines@ipna.csic.es

Supporting information for this article is available on the WWW under http://dx.doi.org/10.1002/ejoc.201101146.

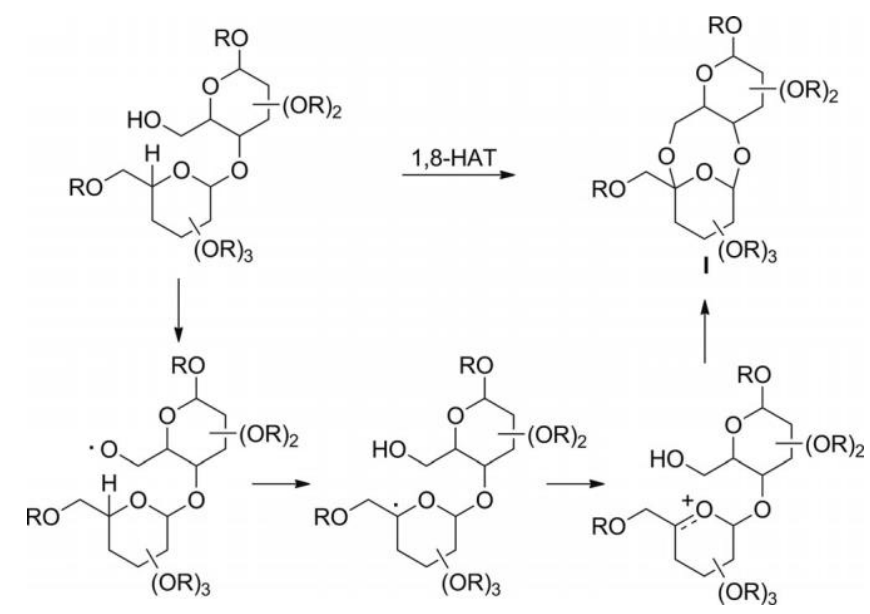

Scheme 1. 1,8-HAT reaction promoted by an O-radical.

\section{Results and Discussion}

The question that immediately arises is whether, and to what extent, this methodology can be applied to N-radicalpromoted reactions considering that to the best of our knowledge this kind of process has never been described previously (Scheme 2). That was the aim of this work, and we report herein the results obtained, which include the first long-distance aminyl-promoted intramolecular HAT reaction and remote functionalization without modification of the remainder of the molecule.

The greater part of the required $N$-sulfonamidate precursors was prepared according to a well-established general protocol by starting from suitably protected disaccharides, as described in the Supporting Information; C-6 primary unprotected alcohols, described in previous articles, ${ }^{[5]}$ were used as starting materials and submitted to a general fourstep protocol. First, conversion into the mesyl derivatives and subsequent nucleophilic substitution with an azide ion took place, then the azides were hydrogenated to the corre- 


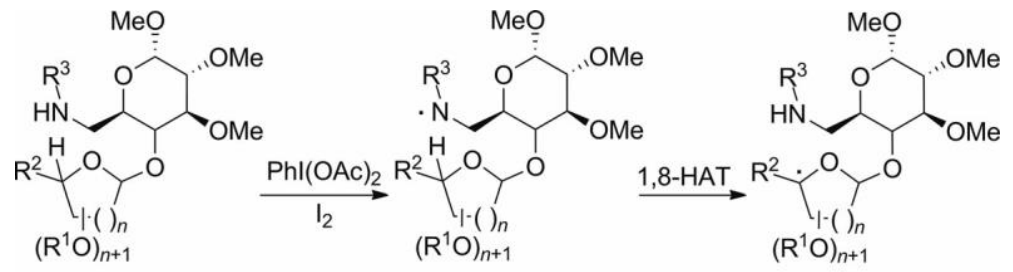

$n=1,2 ; \mathrm{R}^{1}=\mathrm{Me}, \mathrm{Ac} ; \mathrm{R}^{2}=\mathrm{H}, \mathrm{Me}, \mathrm{CH}_{2} \mathrm{OMe}, \mathrm{CH}_{2} \mathrm{OAc} ; \mathrm{R}^{3}=\mathrm{SO}_{2} \mathrm{Ar}$

Scheme 2. 1,8-HAT reaction promoted by N-radicals.

sponding amines, and finally the resulting crude free amines were all treated with the corresponding sulfonyl chloride in the presence of TEA to give the required sulfonamides.

Sulfonamidates were chosen as protecting groups, on the basis of their certain application in amino and amino acid chemistry, ${ }^{[6-8]}$ to avoid oxidation of the amino group during the formation of the iodoamide intermediate and, at the same time, to control the stability of the N-radical during the HAT reaction. In addition, they have been successfully used under our radical conditions to give an assortment of pyrrolidines, as shown by Fan and co-workers. ${ }^{[9]}$ Note also that the carbohydrate structures were selected on the basis of the conclusions drawn from the stereochemical and conformational study of the intramolecular 1,8-HAT reaction when promoted by a primary $6-O$-yl radical. ${ }^{[5 b]}$ Based on these data, we initially prepared the $\alpha-1-$ Rhap $-(1<4)-\alpha-d-$

Table 1. Intramolecular HAT of C-6 sulfonamidyl $\alpha-1-R h a p-(1<4)-\alpha-d-G a l p .^{[a]}$

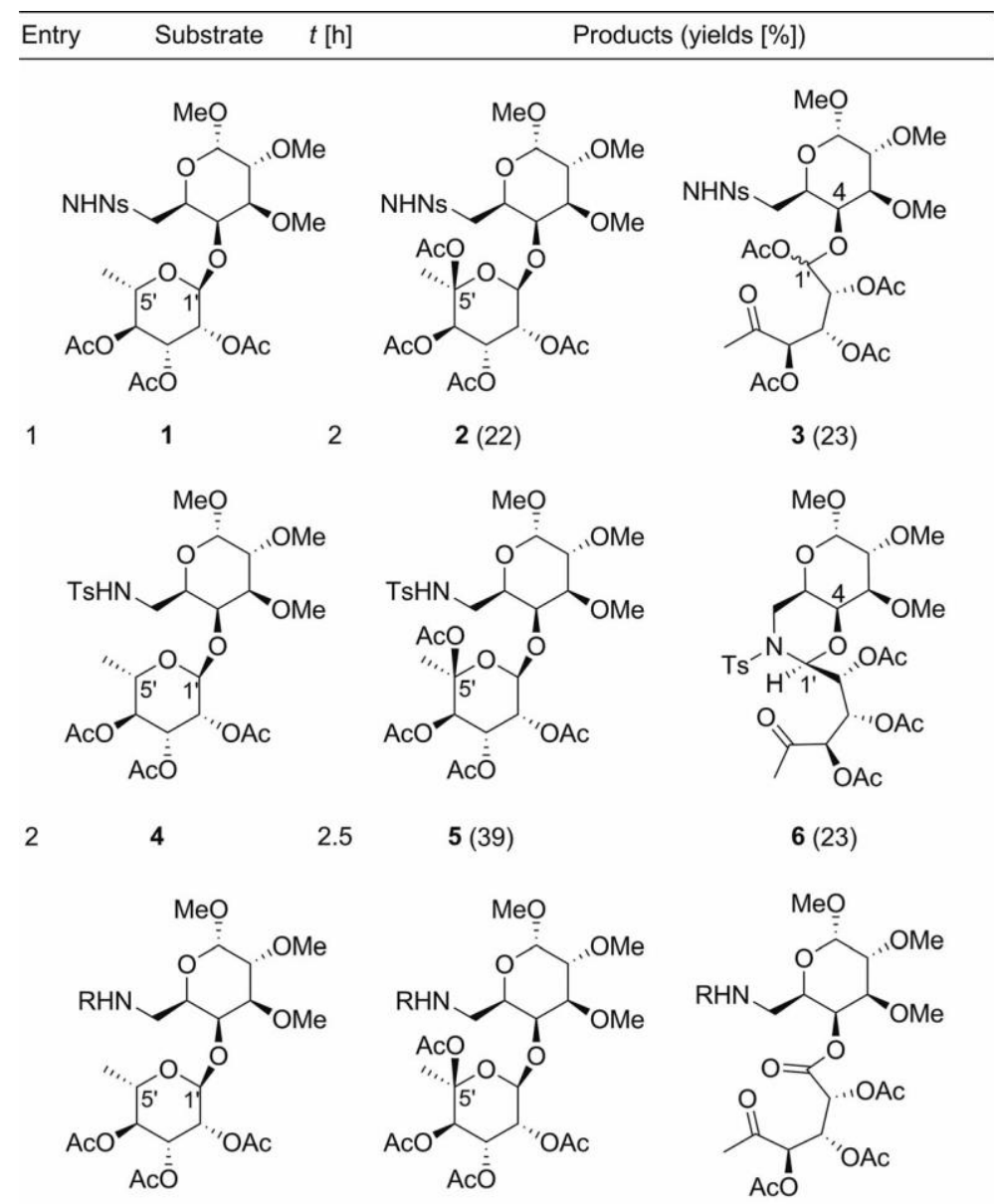

$37 \mathrm{R}=\mathrm{SO}_{2} \mathrm{C}_{6} \mathrm{H}_{4}-p$-OMe $28 \mathrm{R}=\mathrm{SO}_{2} \mathrm{C}_{6} \mathrm{H}_{4}-p-\mathrm{OMe}$ (32) $9 \mathrm{R}=\mathrm{SO}_{2} \mathrm{C}_{6} \mathrm{H}_{4}-p$-OMe (26)

$410 \mathrm{R}=\mathrm{SO}_{2}-2-\mathrm{Py} \quad 511 \mathrm{R}=\mathrm{SO}_{2}-2-\mathrm{Py}(73)$

[a] The sulfonamidyl derivative $(1 \mathrm{mmol})$ in $\mathrm{CH}_{2} \mathrm{Cl}_{2}(20 \mathrm{~mL}$ ) containing (diacetoxyiodo)benzene (DIB; 2.5 mmol) and iodine (1.2 mmol) was irradiated with an $80 \mathrm{~W}$ tungsten filament lamp at room temp. 
Galp disaccharide, which gave the best results in the alkoxyl-promoted abstraction. Derivatives 1, 4, 7, and 10 were successfully prepared and submitted to the intramolecular HAT reaction under the oxidative conditions previously developed by our group (Table 1) with (diacetoxyiodo)benzene and iodine in $\mathrm{CH}_{2} \mathrm{Cl}_{2}$ at room temperature under irradiation with an $80 \mathrm{~W}$ tungsten filament lamp.

We started our study with the radical reactions of the nosyl and tosyl derivatives $\mathbf{1}$ and $\mathbf{4}$, which proceeded smoothly to give, in both cases, a mixture of two compounds with global yields of 45 and $62 \%$, respectively (Table 1, Entries 1 and 2) as a result of the 1,8-HAT reaction. The formation of the acetate derivatives $\mathbf{2}$ and $\mathbf{5}$ shows that the 1,8-HAT reaction occurred, but no cyclization product 1,3,5-dioxazocane was formed, as was reported previously for the O-radical counterpart in which a 1,3,5trioxocane ring I (Scheme 1) was achieved. ${ }^{[5 b]}$ Apparently, a lower nucleophilicity of the sulfonamidate group is responsible for the competitive intermolecular attack of the acetate anion deriving from the reagent. The $(R)$ configuration at $\mathrm{C}^{-5^{\prime}}$ in both products was assigned by the NOE correlations observed between $6^{\prime}-\mathrm{Me}$ and $4^{\prime}-\mathrm{H}$ in a ${ }^{1} \mathrm{C}_{4}$ conformation. Product 3, also derived from the 1,8-HAT reaction, was obtained with $\mathbf{2}$ as an inseparable mixture on ordinary chromatography columns, and may have occurred by a subsequent radical fragmentation of the pyranose ring of II to give, after oxidation of the corresponding C-radical, an oxycarbenium ion III, which is trapped by acetate from the reagent (Scheme 3). Otherwise, probably due to the higher nucleophilicity of the tosylamine relative to the nosyl derivative, in this case, the oxycarbenium ion III was trapped intramolecularly by the tosylamine group to yield a 1,3-oxazolidine cycle in product $\mathbf{6}$. The $(S)$ configuration at $\mathrm{C}-1^{\prime}$ in 6 was assigned on the basis of the NOE correlation observed between $1^{\prime}-\mathrm{H}$ and $4-\mathrm{H}$. In both cases the radical reaction was also tested in the presence of $\mathrm{BF}_{3} \cdot \mathrm{Et}_{2} \mathrm{O}$

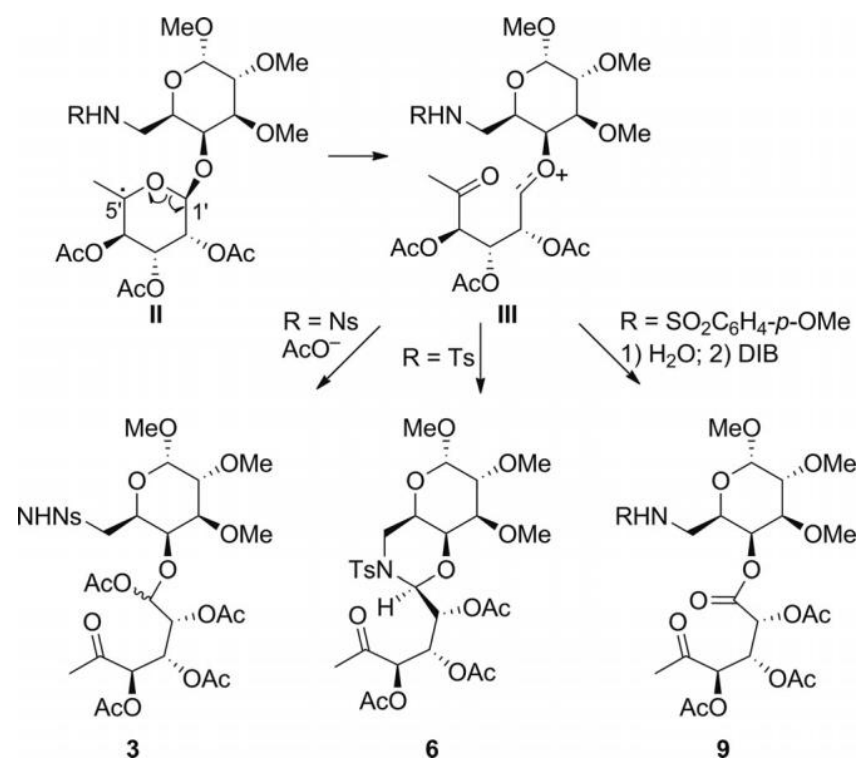

Scheme 3. N-radical-promoted 1,8-HAT and $\beta$-fragmentation. and CSA to promote the intramolecular cyclization to give the 1,3,5-dioxazocane ring, but no changes were observed in the outcome of the process, and only the acetylated derivatives were obtained.

Next we modified the protecting group in an attempt to enhance the nucleophilicity of the amino group with the purpose of obtaining a 1,3,5-dioxazocane substructure, which would constitute a new tricyclic system not previously described in the literature. We then studied the HAT reactions of the ( $p$-methoxyphenyl)sulfonylamine 7 and the 2-pyridinylsulfonylamine 10, which gave 1,8-hydrogen abstraction with global yields of 58 and $73 \%$, respectively (Table 1, Entries 3 and 4). Also in these cases, the corresponding acetyl products $\mathbf{8}$ and 11, deriving from intermolecular attack, were obtained as the major products in a mixture of two, in the case of Entry 3, and as the only product in the case of 2-pyridinylsulfonylamine 11 (Entry 4). Unfortunately, the improvement in the nucleophilicity of the amine with these sulfonyl protecting groups was not sufficient to promote the intramolecular closure, but nevertheless remote functionalization of C-5' through a 1,8-HAT reaction was successful. Again in these cases, the stereochemistry at C-5' was established according to the NOE interactions observed between 6"-Me and 4'-H. The minor product 9 was tentatively formed from the radical fragmentation of the pyranose in II followed by oxidation to an oxycarbenium ion III, which could be trapped by $\mathrm{H}_{2} \mathrm{O}$ molecules to give a hemiacetal intermediate that could be oxidized in the medium to the corresponding $\delta$-keto ester 9 (Table 1, Entry 3; Scheme 3).

Encouraged by these initial results, which may lead to a synthetically useful methodology for the remote functionalization of the C-5' carbon atom, we decided to investigate whether this protocol is exclusive to $\alpha-1-\operatorname{Rhap}-(1<4)-\alpha-\mathrm{d}$ Gal $p$ or, on the contrary, can be extended to other disaccharides that fulfill the stereochemical and conformational requirements for that purpose. With this aim we synthesized $\alpha-\mathrm{d}-\operatorname{Man} p-(1<4)-\alpha-\mathrm{d}-$ Glc $p$ derivatives 12 and $\mathbf{1 4}$ (Table 2, Entries 1 and 2), which were submitted to the oxidative HAT reaction conditions. The results for $\mathbf{1 2}$ showed direct C-5' functionalization via a nine-membered transition state, as observed previously in Table 1 , to give exclusively the acetate derivative $\mathbf{1 3}$ in $52 \%$ yield. The $(S)$ configuration at C-5' was determined on the basis of the NOE interactions observed between $4^{\prime}-\mathrm{H}$ and $6^{\prime}-\mathrm{H}_{\mathrm{a}}$. However, the reaction of the acetate-substituted counterpart $\mathbf{1 4}$ was not successful. Apparently, the electron-withdrawing acetyl groups at C-4" and $\mathrm{C}-6^{\prime}$ can inhibit hydrogen abstraction at C-5', and no reaction was observed, although long reaction times resulted in decomposition of the starting material.

Next, the question arises as to whether the substituent at C-5' in hexopyranose disaccharide models could hinder the intramolecular closure of the amine group and thereby favor intermolecular acetate attack. As a consequence, we prepared $\alpha$-d-Lyx $p-(1<4)-\alpha-\mathrm{d}-\mathrm{Glc} p$ 15, a pentopyranose derivative with no substitution at $\mathrm{C}-5^{\prime}$, and submitted it to the HAT reaction conditions (Table 2, Entry 3). An inseparable mixture of epimers of $\mathbf{1 6}$ was obtained in only $25 \%$ 
Table 2. Intramolecular HAT of C-6 nosyl $\alpha$-d-Man $p-(1<4)-\alpha-d-$ Glc $p, \alpha$-d-Lyxp-(1<4)- $\alpha$-d-Glc $p$, and $\alpha$-d-Araf- $(1<4)-\alpha-\mathrm{d}-\mathrm{Glc} p$. $^{[\mathrm{a}]}$

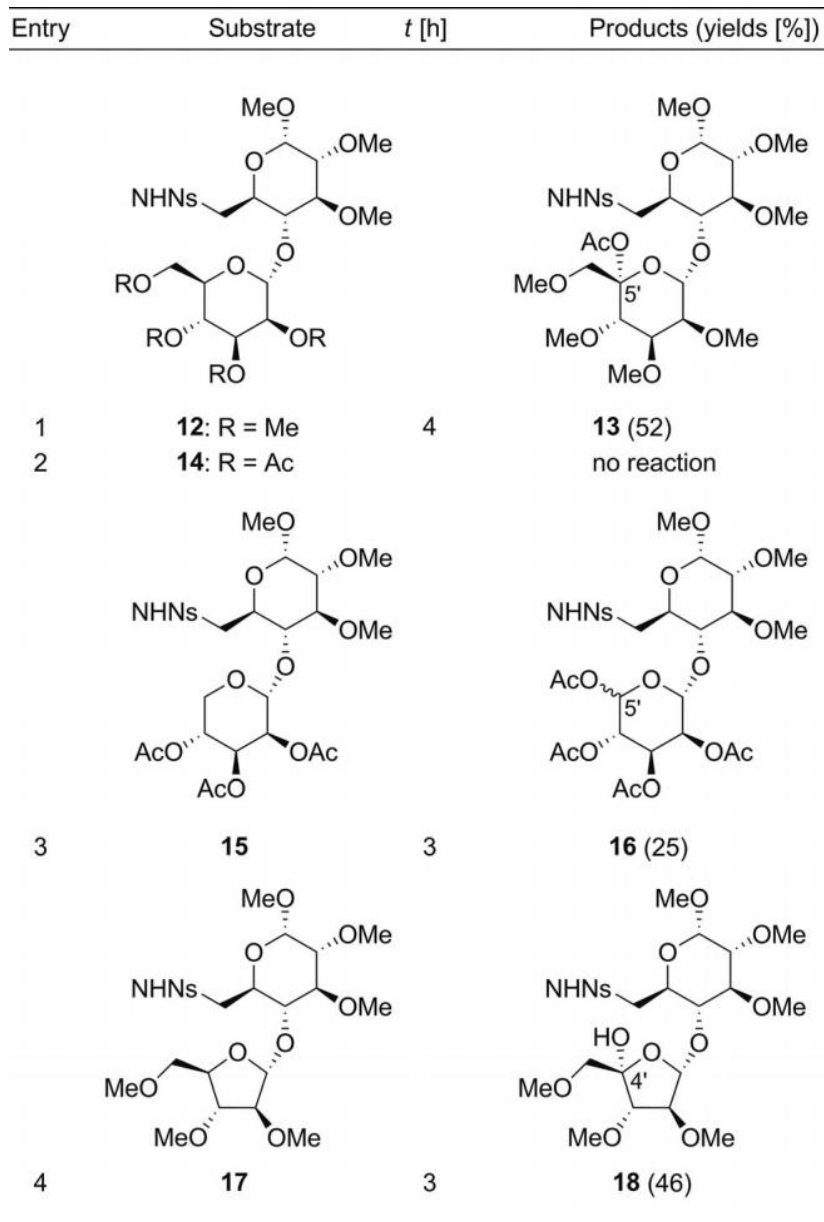

[a] The sulfonamidyl derivative $(1 \mathrm{mmol})$ in $\mathrm{CH} \mathrm{Cl}_{2}(20 \mathrm{~mL})$ containing (diacetoxyiodo)benzene (DIB; $2.5 \mathrm{mmol}$ ) and iodine (1.2 mmol) was irradiated with an $80 \mathrm{~W}$ tungsten filament lamp at room temp.

yield. Destabilization of the oxycarbenium intermediate ion due to the absence of substituents at $\mathrm{C}-5^{\prime}$ could be responsible for the low yield.

Until now, pento- and hexopyranose models have been studied, and the same conclusions as drawn from alkoxyl radicals have been reached, that is, the 1,8-HAT reaction takes place, although lower yields were obtained in these cases.

To complete the study, the pentofuranose models should be revised. Apparently, the sulfonamide group has greater difficulty than the hydroxy group in trapping the oxycarbenium ion, probably due to a combination of steric and electronic factors. It would be worthwhile researching the HAT reaction of a pentofuranose derivative that has less steric hindrance. $\alpha$-d-Araf-( $1<4)-\alpha-d-G l c p \quad 17$ was thus submitted to the standard HAT conditions and amino alcohol 18 was obtained after chromatotron chromatography as the sole product in $46 \%$ yield (Table 2, Entry 4). Traces of the corresponding unstable acetate derivative show that this product was almost completely hydrolyzed to $\mathbf{1 8}$, which can be attributed to the acidity of the reaction medium. The configuration at C-4' was established on the basis of the NOE correlation between $1^{\prime}-\mathrm{H}$ and $5^{\prime}-\mathrm{H}_{2}$.

\section{Conclusions}

To the best of our knowledge, with these examples, we have performed a novel and previously unknown radical 1,8-HAT reaction promoted by $N$-sulfonamidyl radicals. The yields achieved are slightly lower than those previously obtained in the $O$-centered radical studies, and no intramolecular cyclized products were obtained, but the novelty of the process and the ability to promote the remote functionalization of the molecule without modifying the remainder of the disaccharide are highly remarkable and encouraging. The polarity of the amino protecting group may be very important, because the nitrogen atom could act with umpolung reactivity during the reaction, first as an electrophilic $\mathrm{N}$-radical and then as a nucleophile, if the cyclization step was to occur. Further investigations employing more electron-donating protecting groups to support the intramolecular amino cyclization, such as sulfoxides, sulfonamidates, or carbamates, are underway.

\section{Experimental Section}

General Methods: Melting points were determined with a hot-plate apparatus. Optical rotations were measured with a Perkin-Elmer Polarimeter PE-241 at the sodium line at ambient temperature in $\mathrm{CHCl}_{3}$. IR spectra were recorded with a Perkin Elmer 1600/FTIR instrument in $\mathrm{CCl}_{4}$. NMR spectra were recorded with a Bruker AMX 400 spectrometer at $400 \mathrm{MHz}$ for ${ }^{1} \mathrm{H}$ and $100.6 \mathrm{MHz}$ for ${ }^{13} \mathrm{C}$ in $\mathrm{CDCl}_{3}$ in the presence of TMS as internal standard. Mass spectra were recorded with a Waters LCT Premier XE spectrometer by using electrospray ionization (ESI+). Elemental analyses were performed with a Leco TrueSpec Micro instrument. Merck silica gel 60 PF (0.063-0.2 mm) was used for column chromatography. Circular layers of $1 \mathrm{~mm}$ of Merck silica gel 60 PF254 were used with a Chromatotron for centrifugally assisted chromatography. Commercially available reagents and solvents were analytical grade or were purified by standard procedures prior to use. All reactions involving air- or moisture-sensitive materials were carried out under nitrogen. TLC analysis was conducted with a spray of $0.5 \%$ vanillin in $\mathrm{H}_{2} \mathrm{SO}_{4} / \mathrm{EtOH}$ (4:1) and heating until the development of color.

General Procedure for the Oxidative HAT: A solution of $N$-sulfonamidate $(1 \mathrm{mmol})$ in dry $\mathrm{CH}_{2} \mathrm{Cl}_{2}(20 \mathrm{~mL})$ containing DIB $(2.5$ $\mathrm{mmol})$ and iodine $(1.2 \mathrm{mmol})$ under nitrogen was irradiated with one $80 \mathrm{~W}$ tungsten filament lamp at room temperature for $1-6 \mathrm{~h}$. The reaction mixture was then poured into $10 \%$ aqueous $\mathrm{Na}_{2} \mathrm{~S}_{2} \mathrm{O}_{3}$ and extracted with $\mathrm{CH}_{2} \mathrm{Cl}_{2}$, dried with $\mathrm{Na}_{2} \mathrm{SO}_{4}$, concen- trated, and purified by chromatography (hexanes/EtOAc).

Methyl 5-Acetoxy-2,3,4-tri- $O$-acetyl- $\alpha$-L-rhamnopyranosyl-(1<4)6-deoxy-2,3-di- $O$-methyl-6-(4-nitrophenylsulfonylamino)- $\alpha$-D-galactopyranoside (2) and 2,3,4-Tri- $O$-acetyl-6-deoxy-L-lyxo-hexos-5ulose [Methyl 6-Deoxy-2,3-di- $O$-methyl-6-(4-nitrophenylsulfonylamino)- $\alpha$-D-galactopyranoside-4-yl] Acetyl Acetal (3): By starting from $1(91 \mathrm{mg}, 0.13 \mathrm{mmol})$, an inseparable mixture of compounds $\mathbf{2}$ and $\mathbf{3}$ was obtained as a colorless oil (43 mg, $\mathbf{2} / \mathbf{3}=1.4: 1)$ after 
2 h. ${ }^{1} \mathrm{H}$ NMR: $\delta=1.59$ (s, $\left.3 \mathrm{H}\right), 2.02$ (s, $\left.3 \mathrm{H}\right), 2.03$ (s, $\left.6 \mathrm{H}\right), 2.08$ (s, $3 \mathrm{H}), 2.12(\mathrm{~s}, 3 \mathrm{H}), 2.14(\mathrm{~s}, 3 \mathrm{H}), 2.15(\mathrm{~s}, 3 \mathrm{H}), 2.17(\mathrm{~s}, 3 \mathrm{H})$, 2.19 (s, $3 \mathrm{H}$ ), 2.93 (ddd, $J=7.2,7.2,7.2 \mathrm{~Hz}, 1 \mathrm{H}), 3.06$ (ddd, $J=$ 7.4, 7.4, 6.1 Hz, $1 \mathrm{H}), 3.24-3.53(\mathrm{~m}, 8 \mathrm{H}), 3.35$ (s, $3 \mathrm{H}), 3.36$ (s, 3 H), $3.40(\mathrm{~s}, 3 \mathrm{H}), 3.44(\mathrm{~s}, 6 \mathrm{H}), 3.50(\mathrm{~s}, 3 \mathrm{H}), 3.59$ (ddd, $J=10.1$, 3.2, $0 \mathrm{~Hz}, 1 \mathrm{H}), 3.65$ (ddd, $J=8.7,6.4,0 \mathrm{~Hz}, 1 \mathrm{H}), 3.99(\mathrm{~m}, 2 \mathrm{H})$, $4.71(\mathrm{~d}, J=3.2 \mathrm{~Hz}, 1 \mathrm{H}), 4.80(\mathrm{~d}, J=3.7 \mathrm{~Hz}, 1 \mathrm{H}), 5.06(\mathrm{~d}, J=$ $8.5 \mathrm{~Hz}, 2 \mathrm{H}), 5.14(\mathrm{dd}, J=8.2,3.4 \mathrm{~Hz}, 1 \mathrm{H}), 5.23(\mathrm{~m}, 3 \mathrm{H}), 5.41$ $(\mathrm{d}, J=3.2 \mathrm{~Hz}, 1 \mathrm{H}), 5.73(\mathrm{dd}, J=7.0,3.0 \mathrm{~Hz}, 1 \mathrm{H}), 6.27(\mathrm{dd}, J=$ $8.5,5.6 \mathrm{~Hz}, 1 \mathrm{H}), 8.05(\mathrm{~d}, J=9.3 \mathrm{~Hz}, 2 \mathrm{H}), 8.10(\mathrm{~d}, J=9.0 \mathrm{~Hz}, 2$ $\mathrm{H}), 8.35(\mathrm{~d}, J=6.6 \mathrm{~Hz}, 2 \mathrm{H}), 8.36(\mathrm{~d}, J=6.6 \mathrm{~Hz}, 2 \mathrm{H}) \mathrm{ppm}$; signal of $1 \mathrm{H}$ from $\mathrm{NH}$ is missing. An NOE correlation was observed between $6^{\prime}-\mathrm{Me}$ and $4^{\prime}-\mathrm{H}$ in product 2 . The stereochemistry at $\mathrm{C}$ 1 ' in 3 could not be elucidated. ${ }^{13} \mathrm{C}$ NMR: $\delta=20.2\left(\mathrm{CH}_{3}\right), 20.3$ $\left(\mathrm{CH}_{3}\right), 20.4\left(2 \mathrm{CH}_{3}\right), 20.6\left(\mathrm{CH}_{3}\right), 20.7\left(2 \mathrm{CH}_{3}\right), 20.9\left(\mathrm{CH}_{3}\right), 22.0$ $\left(\mathrm{CH}_{3}\right), 26.7\left(\mathrm{CH}_{3}\right), 41.9\left(\mathrm{CH}_{2}\right), 42.6\left(\mathrm{CH}_{2}\right), 55.5\left(\mathrm{CH}_{3}\right), 55.7\left(\mathrm{CH}_{3}\right)$, $57.8\left(\mathrm{CH}_{3}\right), 58.2\left(\mathrm{CH}_{3}\right), 59.0\left(\mathrm{CH}_{3}\right), 59.4\left(\mathrm{CH}_{3}\right), 66.3(\mathrm{CH}), 67.5$ $(\mathrm{CH}), 68.0(\mathrm{CH}), 68.3(\mathrm{CH}), 68.6(\mathrm{CH}), 68.7(\mathrm{CH}), 69.8(\mathrm{CH}), 70.2$ $(\mathrm{CH}), 74.4(\mathrm{CH}), 75.2(\mathrm{CH}), 77.2(\mathrm{CH}), 77.3(\mathrm{CH}), 77.6(\mathrm{CH}), 78.9$ $(\mathrm{CH}), 96.9(2 \mathrm{CH}), 97.9(\mathrm{CH}), 98.2(\mathrm{CH}), 103.9(\mathrm{C}), 124.2(2 \mathrm{CH})$, $124.4(2 \mathrm{CH}), 128.0(2 \mathrm{CH}), 128.3(2 \mathrm{CH}), 145.7(\mathrm{C}), 147.2(\mathrm{C})$, 149.9 (C), 150.1 (C), 167.0 (C), 168.7 (C), 168.9 (C), 169.2 (C),

169.3 (C), 169.4 (2 C), 169.7 (C), 201.7 (C) ppm. IR (CCl ): $\tilde{v}_{4}^{=}$ 2928, 1759, $1215 \mathrm{~cm}^{-1}$. MS $\left(\mathrm{ESI}^{+}\right): \mathrm{m} / z(\%)=759(100)[\mathrm{M}+$ $\mathrm{Na}]^{+}$. HRMS $\left(\mathrm{ESI}^{+}\right)$: calcd. for $\mathrm{C}_{29} \mathrm{H}_{40} \mathrm{~N}_{2} \mathrm{NaO}_{18} \mathrm{~S} 759.1895[\mathrm{M}+$ $\mathrm{Na}]^{+}$; found 759.1890 .

Methyl 5-Acetoxy-2,3,4-tri- $O$-acetyl- $\alpha$-L-rhamnopyranosyl-(1<4)6-deoxy-2,3-di- $O$-methyl-6-(4-methylphenylsulfonylamino)- $\alpha$-D-galactopyranoside (5): By starting from 4 (88.5 mg, $0.14 \mathrm{mmol})$, compound 5 (38 mg, $0.05 \mathrm{mmol}, 39 \%)$ was obtained as a white crystalline solid after 2.5 h. M.p. $55.7-56.0^{\circ} \mathrm{C}(n$-hexane/EtOAc $) . \quad[\alpha]_{\mathrm{D}}=$ $-38.0\left(c=0.050, \mathrm{CHCl}_{3}\right) .{ }^{1} \mathrm{H}$ NMR: $\delta=1.61(\mathrm{~s}, 3 \mathrm{H}), 2.01(\mathrm{~s}, 3$ H), 2.11 (s, $3 \mathrm{H}), 2.13$ (s, $3 \mathrm{H}), 2.15$ (s, $3 \mathrm{H}), 2.42$ (s, $3 \mathrm{H}), 3.20$ $3.25(\mathrm{~m}, 2 \mathrm{H}), 3.35(\mathrm{~s}, 3 \mathrm{H}), 3.38(\mathrm{~m}, 1 \mathrm{H}), 3.43(\mathrm{~m}, 1 \mathrm{H}), 3.44(\mathrm{~s}$, $3 \mathrm{H}), 3.45(\mathrm{~s}, 3 \mathrm{H}), 3.66(\mathrm{~m}, 1 \mathrm{H}), 4.03$ (br. s, $1 \mathrm{H}), 4.73$ (d, $J=$ $2.6 \mathrm{~Hz}, 1 \mathrm{H}), 5.09(\mathrm{dd}, J=8.5,3.7 \mathrm{~Hz}, 1 \mathrm{H}), 5.14(\mathrm{~d}, J=8.5 \mathrm{~Hz}$, $1 \mathrm{H}), 5.27(\mathrm{dd}, J=3.4,3.4 \mathrm{~Hz}, 1 \mathrm{H}), 5.33(\mathrm{~d}, J=3.2 \mathrm{~Hz}, 1 \mathrm{H})$, $5.85(\mathrm{dd}, J=7.7,6.4 \mathrm{~Hz}, 1 \mathrm{H}), 7.29(\mathrm{~d}, J=7.9 \mathrm{~Hz}, 2 \mathrm{H}), 7.79(\mathrm{~d}$, $J=8.2 \mathrm{~Hz}, 2 \mathrm{H}) \mathrm{ppm}$. An NOE correlation was observed between 6"-Me and 4"-H. ${ }^{13} \mathrm{C}$ NMR: $\delta=20.6\left(\mathrm{CH}_{3}\right), 20.7\left(2 \mathrm{CH}_{3}\right), 21.1$ $\left(\mathrm{CH}_{3}\right), 21.5\left(\mathrm{CH}_{3}\right), 21.9\left(\mathrm{CH}_{3}\right), 41.8\left(\mathrm{CH}_{2}\right), 55.4\left(\mathrm{CH}_{3}\right), 58.0\left(\mathrm{CH}_{3}\right)$, $59.0\left(\mathrm{CH}_{3}\right), 66.5(\mathrm{CH}), 67.8(\mathrm{CH}), 68.3(\mathrm{CH}), 68.4(\mathrm{CH}), 73.5(\mathrm{CH})$, $77.5(\mathrm{CH}), 79.1(\mathrm{CH}), 96.4(\mathrm{CH}), 97.8(\mathrm{CH}), 103.9(\mathrm{C}), 126.9(2$ $\mathrm{CH}), 129.6$ (2 CH), 138.3 (C), 143.2 (C), 168.7 (C), 168.9 (2 C), 169.4 (C) ppm. IR $\left(\mathrm{CCl}_{4}\right): \tilde{v}=3312,2933,1754,1219 \mathrm{~cm}^{-1}$. MS $\left(\mathrm{ESI}^{+}\right): m / z(\%)=728(100)[\mathrm{M}+\mathrm{Na}]^{+}$. HRMS $\left(\mathrm{ESI}^{+}\right)$: calcd. for $\mathrm{C}_{30} \mathrm{H}_{43} \mathrm{NNaO}{ }_{16} \mathrm{~S} 728.2200[\mathrm{M}+\mathrm{Na}]^{+}$; found 728.2196. $\mathrm{C}_{30} \mathrm{H}_{43} \mathrm{NO}_{16} \mathrm{~S}$ (705.73): C 51.06, H 6.14, N 1.98, S 4.54; found $\mathrm{C}$ 51.12, H 6.23, N 2.22, S 4.61.

Methyl (1S)-4-O,6- $N$-(2,3,4-Tri-O-acetyl-6-deoxy-L-lyxo-hexos-5ulosylidene)-6-deoxy-2,3-di- $O$-methyl-6-(4-methylphenylsulfonylamino)- $\alpha$-D-galactopyranoside (6): By starting from 4 (88.5 mg, $0.14 \mathrm{mmol})$, compound $6(20 \mathrm{mg}, 0.03 \mathrm{mmol}, 23 \%)$ was obtained as a colorless oil after $2.5 \mathrm{~h} .[\alpha]_{\mathrm{D}}=+16.0\left(c=0.050, \mathrm{CHCl}_{3}\right) .{ }^{1} \mathrm{H}$ NMR: $\delta=2.00$ (s, $3 \mathrm{H}), 2.07$ (s, $3 \mathrm{H}), 2.17$ (s, $3 \mathrm{H}), 2.22$ (s, $3 \mathrm{H})$, $2.42(\mathrm{~s}, 3 \mathrm{H}), 2.89(\mathrm{~m}, 1 \mathrm{H}), 3.05(\mathrm{~m}, 1 \mathrm{H}), 3.33$ (s, $3 \mathrm{H}), 3.40$ (s, 3 H), 3.42-3.55 (m, 2 H), 3.50 (s, $3 \mathrm{H}), 3.95$ (ddd, $J=7.3,7.3,0 \mathrm{~Hz}$, $1 \mathrm{H}), 4.81(\mathrm{~d}, J=3.4 \mathrm{~Hz}, 1 \mathrm{H}), 4.95(\mathrm{dd}, J=6.1,6.1 \mathrm{~Hz}, 1 \mathrm{H})$, $5.29(\mathrm{~d}, J=7.4 \mathrm{~Hz}, 1 \mathrm{H}), 5.37(\mathrm{dd}, J=11.7,2.6 \mathrm{~Hz}, 1 \mathrm{H}), 5.38(\mathrm{~d}$, $J=2.6 \mathrm{~Hz}, 1 \mathrm{H}), 5.76(\mathrm{dd}, J=7.4,2.9 \mathrm{~Hz}, 1 \mathrm{H}), 7.31(\mathrm{~d}, J=$ $7.9 \mathrm{~Hz}, 2 \mathrm{H}), 7.74(\mathrm{~d}, J=8.2 \mathrm{~Hz}, 2 \mathrm{H}) \mathrm{ppm}$. An NOE correlation was observed between $1^{\prime}-\mathrm{H}$ and $4-\mathrm{H} .{ }^{13} \mathrm{C} \mathrm{NMR}: \delta=20.2\left(\mathrm{CH}_{3}\right)$, $20.3\left(\mathrm{CH}_{3}\right), 20.4\left(\mathrm{CH}_{3}\right), 21.5\left(\mathrm{CH}_{3}\right), 26.8\left(\mathrm{CH}_{3}\right), 42.4\left(\mathrm{CH}_{2}\right), 55.7$
$\left(\mathrm{CH}_{3}\right), 57.7\left(\mathrm{CH}_{3}\right), 59.4\left(\mathrm{CH}_{3}\right), 67.4(\mathrm{CH}), 68.5(\mathrm{CH}), 69.7(\mathrm{CH})$, $70.1(\mathrm{CH}), 75.3(\mathrm{CH}), 76.6(\mathrm{CH}), 77.2(\mathrm{CH}), 77.6(\mathrm{CH}), 98.1(\mathrm{CH})$, $127.0(2 \mathrm{CH}), 129.8(2 \mathrm{CH}), 137.2(\mathrm{C}), 143.6(\mathrm{C}), 166.7$ (C), 169.3 (C), $169.8(\mathrm{C}), 201.4(\mathrm{C}) \mathrm{ppm}$. IR $\left(\mathrm{CCl}_{4}\right): \tilde{v}=3389,2932,1757$, $1216 \mathrm{~cm}^{-1}$. MS $\left(\mathrm{ESI}^{+}\right): \mathrm{m} / \mathrm{z}(\%)=668(100)[\mathrm{M}+\mathrm{Na}]^{+}$. HRMS $\left(\mathrm{ESI}^{+}\right)$: calcd. for $\mathrm{C}_{28} \mathrm{H}_{39} \mathrm{NNaO}_{14} \mathrm{~S} 668.2004[\mathrm{M}+\mathrm{Na}]^{+}$; found 668.1989. $\mathrm{C}_{28} \mathrm{H}_{39} \mathrm{NO}_{14} \mathrm{~S}$ (645.68): calcd. C 52.09, H 6.09, N 2.17, S 4.97; found C 52.16, H 6.39, N 2.38, S 5.03.

Methyl 5-Acetoxy-2,3,4-tri- $O$-acetyl- $\alpha$-L-rhamnopyranosyl-( $1<4)$ 6-deoxy-2,3-di- $O$-methyl-6-(4-methoxyphenylsulfonylamino)- $\alpha$-D-galactopyranoside (8): By starting from 7 (184.2 mg, $0.28 \mathrm{mmol})$, compound $8(67 \mathrm{mg}, 0.09 \mathrm{mmol}, 32 \%)$ was obtained as a white crystalline solid after 2 h. M.p. $68.5-69.1{ }^{\circ} \mathrm{C}\left(n\right.$-hexane/EtOAc). $[\alpha]={ }_{\mathrm{D}}$ $-47.5\left(c=0.040, \mathrm{CHCl}_{3}\right)$. ${ }^{1} \mathrm{H}$ NMR: $\delta=1.61(\mathrm{~s}, 3 \mathrm{H}), 2.01(\mathrm{~s}, 3$ $\mathrm{H}), 2.10(\mathrm{~s}, 3 \mathrm{H}), 2.12(\mathrm{~s}, 3 \mathrm{H}), 2.14(\mathrm{~s}, 3 \mathrm{H}), 3.21(\mathrm{dd}, J=7.1$, $7.1 \mathrm{~Hz}, 2 \mathrm{H}), 3.35$ (s, $3 \mathrm{H}), 3.39(\mathrm{dd}, J=4.9,2.8 \mathrm{~Hz}, 2 \mathrm{H}), 3.44(\mathrm{~s}$, $6 \mathrm{H}), 3.66$ (dd, $J=7.7,7.7 \mathrm{~Hz}, 1 \mathrm{H}$ ), 3.85 (s, $3 \mathrm{H}$ ), 4.03 (br. s, 1 $\mathrm{H}), 4.73(\mathrm{~d}, J=2.9 \mathrm{~Hz}, 1 \mathrm{H}), 5.09(\mathrm{dd}, J=8.3,3.8 \mathrm{~Hz}, 1 \mathrm{H}), 5.14$ $(\mathrm{d}, J=8.5 \mathrm{~Hz}, 1 \mathrm{H}), 5.27(\mathrm{dd}, J=3.4,3.4 \mathrm{~Hz}, 1 \mathrm{H}), 5.3(\mathrm{~d}, J=$ $3.2 \mathrm{~Hz}, 1 \mathrm{H}), 5.80(\mathrm{dd}, J=7.2,7.2 \mathrm{~Hz}, 1 \mathrm{H}), 6.95(\mathrm{~d}, J=9.0 \mathrm{~Hz}$, $2 \mathrm{H}), 7.83(\mathrm{~d}, J=9.0 \mathrm{~Hz}, 2 \mathrm{H}) \mathrm{ppm}$. An NOE correlation was observed between 6"-Me and 4"-H. ${ }^{13} \mathrm{C}$ NMR: $\delta=20.5\left(\mathrm{CH}_{3}\right), 20.6$ $\left(2 \mathrm{CH}_{3}\right), 21.1\left(\mathrm{CH}_{3}\right), 21.9\left(\mathrm{CH}_{3}\right), 41.8\left(\mathrm{CH}_{2}\right), 55.4\left(\mathrm{CH}_{3}\right), 55.6$ $\left(\mathrm{CH}_{3}\right), 58.0\left(\mathrm{CH}_{3}\right), 59.0\left(\mathrm{CH}_{3}\right), 66.5(\mathrm{CH}), 67.9(\mathrm{CH}), 68.3(\mathrm{CH})$, $68.5(\mathrm{CH}), 73.5(\mathrm{CH}), 77.6(\mathrm{CH}), 79.2(\mathrm{CH}), 96.4(\mathrm{CH}), 97.9(\mathrm{CH})$, 103.9 (C), $114.1(2 \mathrm{CH}), 129.0$ (2 CH), 132.9 (C), 162.8 (C), 168.7 (2 C), 168.9 (C), 169.3 (C) ppm. IR $\left(\mathrm{CCl}_{4}\right): \tilde{v}=3327,2932,1760$, $1214 \mathrm{~cm}^{-1}$. MS $\left(\mathrm{ESI}^{+}\right): \mathrm{m} / \mathrm{z}(\%)=744(100)[\mathrm{M}+\mathrm{Na}]^{+}$. HRMS $\left(\mathrm{ESI}^{+}\right)$: calcd. for $\mathrm{C}_{30} \mathrm{H}_{43} \mathrm{NNaO}_{17} \mathrm{~S} 744.2149[\mathrm{M}+\mathrm{Na}]^{+}$; found 744.2149. $\mathrm{C}_{30} \mathrm{H}_{43} \mathrm{NO}_{17} \mathrm{~S}$ (721.73): calcd. C 49.93, H 6.01, N 1.94, S 4.44; found C 49.90, H 6.12, N 1.77, S 4.09.

Methyl 4-O-(2,3,4-Tri-O-acetyl-6-deoxy-L-lyxo-hex-5-ulosonoyl)-6deoxy-2,3-di- $O$-methyl-6-(4-methoxyphenylsulfonylamino)- $\alpha$-D-galactopyranoside (9): By starting from 7 (184.2 mg, $0.28 \mathrm{mmol})$, compound 9 (50.4 mg, $0.07 \mathrm{mmol}, 26 \%)$ was obtained as a white crystalline solid after 2 h. M.p. $56.4-57.0{ }^{\circ} \mathrm{C}\left(n\right.$-hexane/EtOAc). $[\alpha]_{\mathrm{D}}=$ $+40.0\left(c=0.030, \mathrm{CHCl}_{3}\right) .{ }^{1} \mathrm{H}$ NMR: $\delta=2.00(\mathrm{~s}, 3 \mathrm{H}), 2.07(\mathrm{~s}, 3$ $\mathrm{H}), 2.17(\mathrm{~s}, 3 \mathrm{H}), 2.21(\mathrm{~s}, 3 \mathrm{H}), 2.86(\mathrm{ddd}, J=6.9,6.9,6.9 \mathrm{~Hz}, 1$ H), $3.02(\mathrm{ddd}, J=13.7,7.8,6.1 \mathrm{~Hz}, 1 \mathrm{H}), 3.33(\mathrm{~s}, 3 \mathrm{H}), 3.39$ (s, 3 $\mathrm{H}), 3.40(\mathrm{dd}, J=9.9,3.6 \mathrm{~Hz}, 1 \mathrm{H}), 3.49(\mathrm{~s}, 3 \mathrm{H}), 3.54(\mathrm{dd}, J=$ 10.1, $3.2 \mathrm{~Hz}, 1 \mathrm{H}), 3.86(\mathrm{~s}, 3 \mathrm{H}), 3.95(\mathrm{dd}, J=7.3,7.3 \mathrm{~Hz}, 1 \mathrm{H})$, $4.80(\mathrm{~d}, J=3.7 \mathrm{~Hz}, 1 \mathrm{H}), 4.94(\mathrm{dd}, J=6.9,6.9 \mathrm{~Hz}, 1 \mathrm{H}), 5.29(\mathrm{~d}$, $J=7.4 \mathrm{~Hz}, 1 \mathrm{H}), 5.36(\mathrm{~d}, J=2.6 \mathrm{~Hz}, 1 \mathrm{H}), 5.38(\mathrm{~d}, J=3.2 \mathrm{~Hz}, 1$ H), $5.76(\mathrm{dd}, J=7.3,3.0 \mathrm{~Hz}, 1 \mathrm{H}), 6.97(\mathrm{~d}, J=9.0 \mathrm{~Hz}, 2 \mathrm{H}), 7.79$ $(\mathrm{d}, J=9.0 \mathrm{~Hz}, 1 \mathrm{H}) \mathrm{ppm} .{ }^{13} \mathrm{C} \mathrm{NMR}: \delta=20.2\left(\mathrm{CH}_{3}\right), 20.3\left(\mathrm{CH}_{3}\right)$, $20.4\left(\mathrm{CH}_{3}\right), 26.7\left(\mathrm{CH}_{3}\right), 42.4\left(\mathrm{CH}_{2}\right), 55.6\left(2 \mathrm{CH}_{3}\right), 57.7\left(\mathrm{CH}_{3}\right), 59.4$ $\left(\mathrm{CH}_{3}\right), 67.4(\mathrm{CH}), 68.6(\mathrm{CH}), 69.8(\mathrm{CH}), 70.1(\mathrm{CH}), 75.4(\mathrm{CH})$, $77.1(\mathrm{CH}), 77.6(\mathrm{CH}), 98.1(\mathrm{CH}), 114.3(2 \mathrm{CH}), 129.1(2 \mathrm{CH}), 131.8$ (C), 163.0 (C), 166.7 (C), 169.2 (2 C), 169.7 (C), 201.3 (C) ppm. IR $\left(\mathrm{CCl}_{4}\right): \tilde{v}=3392,2930,1757,1214,1112 \mathrm{~cm}^{-1}$. MS $\left(\mathrm{ESI}^{+}\right): \mathrm{m} / \mathrm{z}$ $(\%)=700(100)[\mathrm{M}+\mathrm{Na}]^{+}$. HRMS $\left(\mathrm{ESI}^{+}\right)$: calc d. for $\mathrm{C}_{28} \mathrm{H}_{39} \mathrm{NNaO}_{16} \mathrm{~S}: 700.1887[\mathrm{M}+\mathrm{Na}]^{+}$; found 700.1891 . $\mathrm{C}_{28} \mathrm{H}_{39} \mathrm{NO}_{16} \mathrm{~S}$ (677.77): calcd. C 49.63, H 5.80, N 2.07, S 4.73; found C 49.82, H 5.98, N 1.76, S 4.67.

Methyl 5-Acetoxy-2,3,4-tri- $O$-acetyl- $\alpha$-L-rhamnopyranosyl- $(1<4)$ 6-deoxy-2,3-di- $O$-methyl-6-(2-pyridinylsulfonylamino)- $\alpha$-D-galactopyranoside (11): By starting from 10 (95.3 mg, $0.15 \mathrm{mmol})$, compound 11 (75.8 $\mathrm{mg}, 0.11 \mathrm{mmol}, 73 \%$ ) was obtained as a white crystalline solid after 5 h. M.p. $57.1-58.3^{\circ} \mathrm{C}(n$-hexane/EtOAc $) . \quad[\alpha]_{\mathrm{D}}=$ $+4.6\left(c=0.130, \mathrm{CHCl}_{3}\right) .{ }^{1} \mathrm{H}$ NMR: $\delta=1.63(\mathrm{~s}, 3 \mathrm{H}), 2.02(\mathrm{~s}, 3 \mathrm{H})$, $2.10(\mathrm{~s}, 6 \mathrm{H}), 2.13(\mathrm{~s}, 3 \mathrm{H}), 3.33-3.59(\mathrm{~m}, 4 \mathrm{H}), 3.36(\mathrm{~s}, 3 \mathrm{H}), 3.45$ (s, $3 \mathrm{H}), 3.46(\mathrm{~s}, 3 \mathrm{H}), 3.80(\mathrm{dd}, J=7.5,7.5 \mathrm{~Hz}, 1 \mathrm{H}), 4.15$ (br. s, 
$1 \mathrm{H}), 4.75(\mathrm{~d}, J=3.4 \mathrm{~Hz}, 1 \mathrm{H}), 5.14(\mathrm{~s}, 1 \mathrm{H}), 5.14(\mathrm{~m}, 1 \mathrm{H}), 5.28$ $(\mathrm{m}, 1 \mathrm{H}), 5.36(\mathrm{~d}, J=3.4 \mathrm{~Hz}, 1 \mathrm{H}), 6.22(\mathrm{dd}, J=7.0,7.0 \mathrm{~Hz}, 1 \mathrm{H})$, 7.47 (ddd, $J=7.7,4.8,1.1 \mathrm{~Hz}, 1 \mathrm{H}), 7.89$ (ddd, $J=7.7,7.7,1.8 \mathrm{~Hz}$, $1 \mathrm{H}), 8.00(\mathrm{ddd}, J=8.0,1.0,1.0 \mathrm{~Hz}, 1 \mathrm{H}), 8.65(\mathrm{ddd}, J=4.8,1.8$, $1.0 \mathrm{~Hz}, 1 \mathrm{H}) \mathrm{ppm}$. An NOE correlation was observed between $6^{\circ}$ Me and $4^{\circ}-\mathrm{H} .{ }^{13} \mathrm{C}$ NMR: $\delta=20.5\left(\mathrm{CH}_{3}\right), 20.7\left(2 \mathrm{CH}_{3}\right), 21.0\left(\mathrm{CH}_{3}\right)$, $21.9\left(\mathrm{CH}_{3}\right), 42.2\left(\mathrm{CH}_{2}\right), 55.5\left(\mathrm{CH}_{3}\right), 58.0\left(\mathrm{CH}_{3}\right), 59.0\left(\mathrm{CH}_{3}\right), 66.5$ $(\mathrm{CH}), 68.3(2 \mathrm{CH}), 68.4(\mathrm{CH}), 73.8(\mathrm{CH}), 77.6(\mathrm{CH}), 79.1(\mathrm{CH})$, $96.5(\mathrm{CH}), 97.8(\mathrm{CH}), 103.9(\mathrm{C}), 121.9(\mathrm{CH}), 126.4(\mathrm{CH}), 137.9$ $(\mathrm{CH}), 149.8(\mathrm{CH}), 158.2(\mathrm{C}), 168.7(\mathrm{C}), 168.6(\mathrm{C}), 168.9(\mathrm{C}), 169.4$ (C) ppm. IR $\left(\mathrm{CCl}_{4}\right): \tilde{v}=3315,2931,1757,1215 \mathrm{~cm}^{-1}$. MS $\left(\mathrm{ESI}^{+}\right)$: $m / z(\%)=715(100)[\mathrm{M}+\mathrm{Na}]^{+}$. HRMS $\left(\mathrm{ESI}^{+}\right)$: calcd. for $\mathrm{C}_{28} \mathrm{H}_{40} \mathrm{~N}_{2} \mathrm{NaO}_{16} \mathrm{~S} 715.1996[\mathrm{M}+\mathrm{Na}]^{+}$; found 715. 1987 $\mathrm{C}_{28} \mathrm{H}_{40} \mathrm{~N}_{2} \mathrm{O}_{16} \mathrm{~S}$ (692.69): calcd. C 48.55, H 5.82, N 4.04, S 4.63; found C 48.35, H 5.62, N 4.31, S 4.42 .

Methyl 5-Acetoxy-2,3,4,6-tetra- $O$-methyl- $\alpha$-D-mannopyranosyl(1<4)-6-deoxy-2,3-di- $O$-methyl-6-(4-nitrophenylsulfonylamino)- $\alpha$-Dglucopyranoside (13): Starting from $12(43.8 \mathrm{mg}, 0.07 \mathrm{mmol})$, compound 13 ( $25 \mathrm{mg}, 0.04 \mathrm{mmol}, 52 \%$ ) was obtained as an amorphous solid after 4 h. $[\alpha]_{\mathrm{D}}=+2.2\left(c=0.09, \mathrm{CHCl}_{3}\right) .{ }^{1} \mathrm{H}$ NMR: $\delta=2.16$ $(\mathrm{dd}, J=9.5,3.4 \mathrm{~Hz}, 1 \mathrm{H}), 2.16(\mathrm{~s}, 3 \mathrm{H}), 3.12(\mathrm{dd}, J=10.1,9.0 \mathrm{~Hz}$, $1 \mathrm{H}), 3.24(\mathrm{~s}, 3 \mathrm{H}), 3.27$ (s, $3 \mathrm{H}), 3.27-3.35(\mathrm{~m}, 3 \mathrm{H}), 3.42(\mathrm{~s}, 3 \mathrm{H})$, $3.46(\mathrm{~s}, 3 \mathrm{H}), 3.47(\mathrm{~s}, 3 \mathrm{H}), 3.48(\mathrm{~s}, 3 \mathrm{H}), 3.52(\mathrm{~s}, 3 \mathrm{H}), 3.56-3.60$ $(\mathrm{m}, 2 \mathrm{H}), 3.74-3.78(\mathrm{~m}, 3 \mathrm{H}), 4.02(\mathrm{~d}, J=3.7 \mathrm{~Hz}, 1 \mathrm{H}), 4.09(\mathrm{~d}, J$ $=8.7 \mathrm{~Hz}, 1 \mathrm{H}), 5.15(\mathrm{~d}, J=8.2 \mathrm{~Hz}, 1 \mathrm{H}), 6.66(\mathrm{dd}, J=6.5,6.5 \mathrm{~Hz}$, $1 \mathrm{H}), 8.20(\mathrm{~d}, J=9.0 \mathrm{~Hz}, 2 \mathrm{H}), 8.29(\mathrm{~d}, J=9.0 \mathrm{~Hz}, 2 \mathrm{H}) \mathrm{ppm}$. An NOE correlation was observed between $4^{\prime}-\mathrm{H}$ and $6^{\prime}-\mathrm{H}_{\mathrm{a}} \cdot{ }^{13} \mathrm{C}$ NMR: $\delta=22.3\left(\mathrm{CH}_{3}\right), 42.9\left(\mathrm{CH}_{2}\right), 55.3\left(\mathrm{CH}_{3}\right), 58.1\left(\mathrm{CH}_{3}\right), 58.6\left(\mathrm{CH}_{3}\right)$, $59.4\left(2 \mathrm{CH}_{3}\right), 59.6\left(\mathrm{CH}_{3}\right), 61.2\left(\mathrm{CH}_{3}\right), 68.9(\mathrm{CH}), 70.2\left(\mathrm{CH}_{2}\right), 74.0$ $(\mathrm{CH}), 76.1(\mathrm{CH}), 76.8(\mathrm{CH}), 77.2(\mathrm{CH}), 81.7(\mathrm{CH}), 82.2(\mathrm{CH}), 96.7$ $(\mathrm{CH}), 98.4(\mathrm{CH}), 104.4(\mathrm{C}), 123.3(2 \mathrm{CH}), 128.5(2 \mathrm{CH}), 149.3(\mathrm{C})$, $149.4(\mathrm{C}), 170.3$ (C) ppm. IR (film): $\tilde{v}=3301,2933,1733$, $1347 \mathrm{~cm}^{-1}$. MS $\left(\mathrm{ESI}^{+}\right): \mathrm{m} / z(\%)=705(100)[\mathrm{M}+\mathrm{Na}]^{+}$. HRMS $\left(\mathrm{ESI}^{+}\right.$): calcd. for $\mathrm{C}_{27} \mathrm{H}_{42} \mathrm{~N}_{2} \mathrm{NaO}_{16} \mathrm{~S} 705.2153[\mathrm{M}+\mathrm{Na}]^{+}$; found 705.2157. $\mathrm{C}_{2} \mathrm{H}_{4} \mathrm{~N}_{2} \mathrm{O}$ S (682.69): calcd. C 47.50, H 6.20, N 4.10, S 4.70; found C 47.52, H 6.09, N 3.83, S 4.54.

Methyl 5-Acetoxy-2,3,4-tri- $O$-acetyl- $\alpha$-D-lyxopyranosyl-(1<4)-6deoxy-2,3-di- $O$-methyl-6-(4-nitrophenylsulfonylamino)- $\alpha$-D-glucopyranoside (16): By starting from 15 (44.2 $\mathrm{mg}, 0.07 \mathrm{mmol})$, compound $16\left(12.1 \mathrm{mg}, 0.02 \mathrm{mmol}, 25 \%, 5^{\prime} S / 5^{\circ} R, 4.3: 1\right)$ was obtained as a mixture of epimers and as a colorless oil after $3 \mathrm{~h} .{ }^{1} \mathrm{H}$ NMR [only the major $\left(5^{\prime} S\right)$ isomer is described]: $\delta=2.04(\mathrm{~s}, 3 \mathrm{H}), 2.09(\mathrm{~s}, 3 \mathrm{H})$, $2.13(\mathrm{~s}, 3 \mathrm{H}), 2.15(\mathrm{~s}, 3 \mathrm{H}), 2.81(\mathrm{dd}, J=9.5,3.4 \mathrm{~Hz}, 1 \mathrm{H})$,

3.31-3.64 (m, $5 \mathrm{H}), 3.37$ (s, $3 \mathrm{H}), 3.39$ (s, $3 \mathrm{H}), 3.55$ (s, $3 \mathrm{H}), 4.55$ $(\mathrm{d}, J=3.4 \mathrm{~Hz}, 1 \mathrm{H}), 5.26(\mathrm{~m}, 1 \mathrm{H}), 5.29(\mathrm{~d}, J=7.4 \mathrm{~Hz}, 1 \mathrm{H}), 5.30$ $5.32(\mathrm{~m}, 2 \mathrm{H}), 5.85(\mathrm{~d}, J=6.9 \mathrm{~Hz}, 1 \mathrm{H}), 8.13(\mathrm{~d}, J=8.7 \mathrm{~Hz}, 2 \mathrm{H})$, $8.34(\mathrm{~d}, J=8.7 \mathrm{~Hz}, 2 \mathrm{H}) \mathrm{ppm}$; the signal of $1 \mathrm{H}$ from $\mathrm{NH}$ is missing. ${ }^{13} \mathrm{C}$ NMR [only the major $\left(5^{\circ} \mathrm{S}\right)$ isomer is described]: $\delta=$ $20.6\left(\mathrm{CH}_{3}\right), 20.7\left(2 \mathrm{CH}_{3}\right), 21.0\left(\mathrm{CH}_{3}\right), 43.9\left(\mathrm{CH}_{2}\right), 55.6\left(\mathrm{CH}_{3}\right), 58.7$ $\left(\mathrm{CH}_{3}\right), 61.1\left(\mathrm{CH}_{3}\right), 67.8(\mathrm{CH}), 67.9(\mathrm{CH}), 68.4(\mathrm{CH}), 68.6(\mathrm{CH})$, $78.0(\mathrm{CH}), 82.2(\mathrm{CH}), 82.3(\mathrm{CH}), 89.9(\mathrm{CH}), 97.0(\mathrm{CH}), 98.6(\mathrm{CH})$, $123.9(2 \mathrm{CH}), 128.6(2 \mathrm{CH}), 146.9(\mathrm{C}), 149.9(\mathrm{C}), 169.3(\mathrm{C}), 169.6$ (2 C), 169.9 (C) ppm. IR $\left(\mathrm{CCl}_{4}\right): \tilde{v}=3357,2933,1759,1219 \mathrm{~cm}^{-1}$. MS $\left(\mathrm{ESI}^{+}\right): \mathrm{m} / \mathrm{z}(\%)=745(100)[\mathrm{M}+\mathrm{Na}]^{+}$. HRMS $\left(\mathrm{ESI}^{+}\right)$: calcd. for $\mathrm{C}_{28} \mathrm{H}_{38} \mathrm{~N}_{2} \mathrm{NaO}_{18} \mathrm{~S} 745.1738[\mathrm{M}+\mathrm{Na}]^{+}$; found 745.1736 .

Methyl 4-Hydroxy-2,3,5-tri- $O$-methyl- $\alpha$-D-arabinofuranosyl-(1<4)6-deoxy-2,3-di- $O$-methyl-6-(4-nitrophenylsulfonylamino)- $\alpha$-D-glucopyranoside (18): By starting from $17(48.7 \mathrm{mg}, 0.08 \mathrm{mmol})$, compound 18 (24 mg, $0.04 \mathrm{mmol}, 46 \%)$ was obtained as colorless oil after 3 h. $[\alpha]_{\mathrm{D}}=+96.3\left(c=0.320, \mathrm{CHCl}_{3}\right) .{ }^{1} \mathrm{H} \mathrm{NMR}: \delta=2.88(\mathrm{dd}$, $J=9.5,3.4 \mathrm{~Hz}, 1 \mathrm{H}), 3.30-3.58(\mathrm{~m}, 7 \mathrm{H}), 3.35(\mathrm{~s}, 3 \mathrm{H}), 3.41(\mathrm{~s}, 3$ H), 3.54 (s, $3 \mathrm{H}), 3.46$ (s, $3 \mathrm{H}), 3.53$ (s, $3 \mathrm{H}), 3.54$ (s, $3 \mathrm{H}), 3.72$ (d,
$J=4.8 \mathrm{~Hz}, 1 \mathrm{H}), 3.82(\mathrm{dd}, J=4.6,2.5 \mathrm{~Hz}, 1 \mathrm{H}), 4.60(\mathrm{~d}, J=$ $3.7 \mathrm{~Hz}, 1 \mathrm{H}), 5.37(\mathrm{~d}, J=2.4 \mathrm{~Hz}, 1 \mathrm{H}), 6.03(\mathrm{dd}, J=6.8,6.8 \mathrm{~Hz}$, $1 \mathrm{H}), 8.11(\mathrm{~d}, J=9.0 \mathrm{~Hz}, 2 \mathrm{H}), 8.33(\mathrm{~d}, J=9.0 \mathrm{~Hz}, 2 \mathrm{H}) \mathrm{ppm}$; the signal of $1 \mathrm{H}$ from $\mathrm{OH}$ is missing. An NOE correlation was observed between $1^{\prime}-\mathrm{H}$ and $5^{\prime}-\mathrm{H}_{2} .{ }^{13} \mathrm{C}$ NMR: $\delta=44.4\left(\mathrm{CH}_{2}\right), 55.3$ $\left(\mathrm{CH}_{3}\right), 57.9\left(\mathrm{CH}_{3}\right), 58.6\left(\mathrm{CH}_{3}\right), 59.0\left(\mathrm{CH}_{3}\right), 59.6\left(\mathrm{CH}_{3}\right), 60.8\left(\mathrm{CH}_{3}\right)$, $68.7(\mathrm{CH}), 75.0\left(\mathrm{CH}_{2}\right), 76.2(\mathrm{CH}), 82.4(\mathrm{CH}), 82.7(\mathrm{CH}), 83.9(\mathrm{CH})$, $88.6(\mathrm{CH}), 97.1(\mathrm{CH}), 103.7(\mathrm{C}), 106.4(\mathrm{CH}), 124.0(2 \mathrm{CH}), 128.5$ $(2 \mathrm{CH}), 147.1(\mathrm{C}), 149.8(\mathrm{C}) \mathrm{ppm}$. IR $\left(\mathrm{CCl}_{4}\right): \tilde{v}=3489,3290,2934$, 1534, $1348 \mathrm{~cm}^{-1}$. MS $\left(\mathrm{ESI}^{+}\right): \mathrm{m} / z(\%)=619(100)[\mathrm{M}+\mathrm{Na}]^{+}$. HRMS $\left(\mathrm{ESI}^{+}\right)$: calcd. for $\mathrm{C}_{23} \mathrm{H}_{36} \mathrm{~N}_{2} \mathrm{NaO}_{14} \mathrm{~S} 619.1785[\mathrm{M}+\mathrm{Na}]^{+}$; found 619.1793. $\mathrm{C}_{23} \mathrm{H}_{36} \mathrm{~N}_{2} \mathrm{O}_{14} \mathrm{~S}$ (596.60): calcd. C 46.30, H 6.08, N 4.70, S 5.37; found C 46.42, H 6.37, N 4.91, S 5.09.

Supporting Information (see footnote on the first page of this article): Complete description of the experimental details of precursors and analytical data for all new compounds.

\section{Acknowledgments}

This work was supported by the Gobierno de Canarias (Research Program SolSubC200801000192) and the Ministerio de Ciencia e Innovación (Research Program CTQ2010-18244), co-financed by the Fondo Europeo de Desarrollo Regional (FEDER). I. P.-M. thanks the Ministerio de Ciencia e Innovación for support (Research Program PIE 200930I138).

1 For recent reviews, see: a) J. M. Tanko, Annu. Rep. Prog. Chem., Sect. B 2006, 102, 247-268; b) J. Robertson, J. Pillai, R. K. Lush, Chem. Soc. Rev. 2001, 30, 94-103; c) L. Feray, N. Kuznersov, P. Renaud in Radicals in Organic Synthesis (Eds.: P. Renaud, M.P. Sibi), Wiley-VCH, Weinheim, 2001, vol. 2, pp. 246-278; d) G. Majetich, Tetrahedron 1995, 51, 7095-7129.

2 a) A. Martín, I. Pérez-Martín, L. M. Quintanal, E. Suárez, Tetrahedron Lett. 2008, 49, 5179-5181; b) C. G. Francisco, R. Freire, A. J. Herrera, I. Pérez-Martín, E. Suárez, Tetrahedron 2007, 63, 8910-8920; c) C. G. Francisco, A. J. Herrera, E. Suárez, J. Org. Chem. 2002, 67, 7439-7445; d) R. L. Dorta, A. Martín, J. A. Salazar, E. Suárez, J. Org. Chem. 1998, 63, 22512261; e) A. Martín, J. A. Salazar, E. Suárez, J. Org. Chem. 1996, 61, 3999-4006.

3 a) A. Martín, I. Pérez-Martín, E. Suárez, Tetrahedron 2009, 65, 6147-6155; b) C. G. Francisco, A. J. Herrera, A. Martín, I. Pérez-Martín, E. Suárez, Tetrahedron Lett. 2007, 48, 63846388; c) A. Martín, I. Pérez-Martín, E. Suárez, Org. Lett. 2005, 7, 2027-2030; d) C. G. Francisco, A. J. Herrera, E. Suárez, J. Org. Chem. 2003, 68, 1012-1017.

4 Only a few examples of intramolecular HAT reactions that proceed via eight- or higher-membered transition states have been reported, limiting their applications to appropriate skeletons such as steroids; for example, see: a) K. Orito, M. Ohto, N. Sugawara, S. Suginome, Tetrahedron Lett. 1990, 31, 5921-5924; b) K. Orito, S. Satoh, H. Suginome, J. Chem. Soc., Chem. Commun. 1989, 1829-1831; c) R. L. Wife, D. Prezant, R. Breslow, Tetrahedron Lett. 1976, 17, 517-520.

5 a) E. I. León, A. Martín, I. Pérez-Martín, L. M. Quintanal, E. Suárez, Eur. J. Org. Chem. 2010, 5248-5262; b) C. G. Francisco, A. J. Herrera, A. R. Kennedy, A. Martín, D. Melián, I. PérezMartín, L. M. Quintanal, E. Suárez, Chem. Eur. J. 2008, 14, 10369-10381.

6 a) T. Greene, P. G. M. Wuts, Protective Groups in Organic Synthesis, 4th ed., Wiley, Hoboken, NJ, 2007, pp. 851-868; b) P. J. Kocienski, Protecting Groups, 3rd ed., Georg Thieme, Stuttgart, 2005, pp. 543-558, and references cited therein.

7 For reviews of nosyl chemistry, see: a) T. Kan, T. J. Fukuyama, J. Synth. Org. Chem., Jpn. 2001, 59, 779-789; b) T. Kan, T. Fukuyama, Chem. Commun. 2004, 353-359, and references 
cited therein; c) for nosyl as a protecting group in peptides, see: A. Leggio, M. L. Di Gioia, F. Perri, A. Liguori, Tetrahedron 2007, 63, 8164-8173.

[8] a) For the 2-(1,3-dioxan-2-yl)ethylsulfonyl group (Dios), see: I. Sakamoto, N. Izumi, T. Yamada, T. Tsunoda, Org. Lett. 2006, $8,71-74 ; \mathrm{b}$ ) for the $\beta$-trimethylsilylethylsulfonyl group (SES), see: P. Ribière, V. Declerck, J. Martinez, F. Lamaty, Chem. Rev. 2006, 106, 2249-2269; c) for the ortho-anisylsulfonyl group, see: R. R. Milburn, V. Snieckus, Angew. Chem. 2004, 116, 910; Angew. Chem. Int. Ed. 2004, 43, 892-894; d) for the 2,2,5,7,8- pentamethylchromanyl-6-sulfonyl (Pmc) group, see: J. Green, O. M. Ogunjobi, R. Ramage, A. S. J. Stewart, S. McCurdy, R. Noble, Tetrahedron Lett. 1988, 29, 4341-4344.

[9] a) R. Fan, D. Pu, F. Wen, J. Wu, J. Org. Chem. 2007, 72, 89948997 ; b) for intermolecular HAT promoted by N-radicals under these conditions, see: R. Fan, W. Li, D. Pu, L. Zhang, Org. Lett. 2009, 11, 1425-1428.

Received: August 4, 2011 Published Online: October 25, 2011 